\title{
Editorial
}

\section{Lung cancer: Is biopsy mandatory}

"To be, or not to be: That is the question" - (Act III, Scene I).

-Hamlet. William Shakespeare

This paradox can present itself, to the clinician, on detection of asymptomatic lung lesion on routine screening procedure - is it malignancy or infectious process? A pulmonary nodule in a 40-year-old nonsmoker male with rounded margins compared to a 60-year-old elderly male, heavy smoker, with spiculated margins and pleural retraction are two extreme ends of routine clinical scenarios. Is biopsy mandated? This causes much consternation in insurance-funded health-care systems that demand proof of cost-effectiveness in patients who are diagnosed with these lesions but have not yet succumbed to full-blown malignancy. As medical science makes an epochal progress in unraveling intricate molecular pathogenesis, this question is likely to face a stronger scrutiny. A perfect closure needs a resolution from the rigorous scientific approach with a constructive mindset. This has become paramount in view of increasing incidence worldwide with a direct correlation to smoking (tobacco epidemic) and pollution due to urbanization with numbers projected to increase to over 3 million by 2035. ${ }^{[1]}$ Over 1.6 million patients having succumbed to this deadly scourge and is fast becoming a major public health concern worldwide. ${ }^{[2]}$ In the molecular era, our understanding has evolved to the identification of "driver mutation" (based on genotype); this is reflected on the difference in incidence rates when molecular epidemiology is accounted for. ${ }^{[3]}$ For example, epidermal growth factor receptor (EGFR) mutations are different in "never-smokers" versus "smokers" (in addition to known factors causing molecular heterogeneity such as geographical regions, race, and ethnicity). A direct effect is on the difference in clinical outcomes. Genome-wide association studies can probe this question deeply to eke out answers. Despite the growing incidence, interestingly, age-adjusted mortality rates are declining for developed countries (roughly 1\%/year, likely due to effective screening of susceptible population) but an observable increase in incidence and mortality in women due to similar patterns of modifiable behavioral risk factors such as smoking. ${ }^{[4]}$ This emphasizes the role of adequate cost-effective and reliable screening of susceptible population in addition to prevention by active smoking cessation programs. The role of proper histopathological diagnosis for lung masses cannot be overstated.
A number of procedures have evolved to detect lung masses. The emphasis is on minimally invasive techniques to maximize the yield of ratio of tumor tissue to normal cells for better pathological characterization..$^{[5]}$ It involves a multidisciplinary effort to localize lung masses with minimal morbidity. Autofluorescence bronchoscopy, radial endobronchial endoscopy and ultrasound with mini probes and integrated guided sheaths, electromagnetic navigation bronchoscopy, virtual bronchoscopy navigation, and confocal microendoscopy are some of the newer methods to approach patients. These techniques have evolved from sputum cytology screening to the present day autofluorescence bronchoscopy which detects dysplastic changes with different colors for easy discrimination. Confocal microendoscopy shows up a cross-section of the cell layers generated in two dimensions. ${ }^{[6]}$ This is done by algorithmic generation and processing of raw imaging data in real time. Incorporation of narrow band imaging acts complementarily to these established methodologies. All these are helped by "rapid-on-site-cytology" to guide this process.

Biological diversity of lung cancers has long been recognized. This has led to demands for more tissue at the time of initial presentation and during the follow-up after definitive treatment for any residual lesion. However, it comes with the caveat of increasing risk of iatrogenic effects (vide infra) making it practically difficult. Concomitantly, the clinicians miss out additional spatial information about the evolution of tumor biology dynamics through the time course. In the current era of personalized medicine, it aims to improve "tumor hit" (through targeted therapies) with minimal "collateral damage" to the normal tissues. ${ }^{[7]}$ Bevacizumab and antifolate pemetrexed (acting on a nonsquamous variant of nonsmall lung cancer) have been firmly established in the armamentarium against lung cancer. EGFR mutations (in exons 19 and 21) and echinoderm microtubule-associated protein-like 4 and anaplastic lymphoma kinase (EML4-ALK) rearrangements have been identified in exquisite detail (in about $10 \%$ of nonsmall cell lung cancer [NSCLC] tumors). ${ }^{[8]}$ Newer molecules such as gefitinib, erlotinib (acting as EGFR0 tyrosine kinase inhibitor), afatinib (ErbB inhibitor), crizotinib (ALK and MET inhibitor) have either been approved as first-/second-line agents or undergoing prospective trials (alone or as combination 
regimes). ${ }^{[9]}$ Therefore, it becomes imperative to define and overcome the challenges posed by limitations on adequate tissue sampling. ${ }^{[10]}$

Peripheral lung masses are increasingly being diagnosed as adenocarcinomas (mucin production on hematoxylin and eosin staining). Pneumocytic markers, thyroid transcription factor-1, and Napsin A on immunohistochemistry (IHC) are pathognomonic in over $85 \%$ of cases even in small tissue samples. Keratinization and formation of intercellular bridges herald squamous cell carcinoma. Effective IHC analysis requires the use of "antibody cocktails" or "double labeling," for limited tissue samples to identify poorly differentiated carcinomas and exclusion of metastatic disease wherein tissue architecture may not be appreciated well. ${ }^{[11]}$ Increasing amount of tissue, whether obtained by fine-needle aspiration or through core biopsy, needs to be processed and stored for further molecular studies. Hence, adjunct diagnostic correlates need to be established where biopsy is not feasible.

The role of high-resolution contrast-enhanced computed tomography (HRCT) scan has been firmly established. There is little consensus on the serial imaging of isolated nodules $(<3 \mathrm{~cm})$ due to heterogeneity in retrospective data, interobserver variation due to differences in techniques of acquisition, image reconstruction, different methods of assessment (manual versus volumetric using software approaches), and/or use of dynamic contrast CT scan. ${ }^{[12]}$ This issue has been exacerbated by serial CT imaging for high-risk individuals (smokers) in lung cancer screening protocols. While attractive in theory, its cost-effectiveness to prevent mortality has not been established.

This presents itself as a Hobson's dilemma-repeated intervention (biopsy/surgical approaches followed by adjuvant therapy as dictated by existing guidelines) versus observation alone for isolated pulmonary nodules detected on screening. Two major unanswered questions remain: At what time intervention needs to be done and how aggressive the surveillance ought to be. Validated models of predictors of malignancy incorporating the age, smoking risk, diameter, spiculation, and location help differentiate between benign versus malignant nodules. ${ }^{[13]}$ Volume doubling time is time taken for a spherical nodule to grow double in size. A longer lag time exists for ground glass nodules than spiculated ones, for which the volume doubles to twice the size in susceptible patients (18-131 days on an average) for any delay in definitive treatment. ${ }^{[14]}$ However, these data are gleaned from retrospective studies that are prone to observer bias. The tipping point for unresectability is not known during the period of observation. Despite this, imaging remains the cornerstone for diagnosis and is robust enough to identify suspicious nodules to follow them up for histological diagnosis.

The risk of provoking the potential iatrogenic effects is sizable. A surgical resection is likely to cause persistent air leak $(3 \%-5 \%)$, pneumonia, and worsening of compromised lung function. A bronchoscopic (or CT-guided) procedure can cause pneumothorax $(2 \%-4 \%)$ and high rate of false-negative rates $(30 \%-70 \%)$; in that case, a surgical biopsy is the recourse. Radiological surveillance extracts a huge psychological toll of uncertainty (in addition, subjecting affected individuals to increased amounts of radiation). ${ }^{[15]}$ More importantly, additional tissue is required for molecular tests and analysis during different time course of intervals to maximize the therapeutic ratio. A sparse paraffin-embedded tissue leads to deamination of cytosine $(\mathrm{C}>\mathrm{T})$ on the molecular testing, compounds the problem further. ${ }^{[16]}$

Functional imaging using 18-flourodeoxyglucose positron emission tomography (FDG-PET) has emerged as a supplementary modality to HRCT. Arguably, concerns remain for its utility for interpretation in infectious coexisting with malignancy in endemic areas. In a meta-analysis, FDG-PET had a lower average adjusted specificity (16\%) compared with nonendemic regions $(61 \%$ vs. $77 \%$ with adjusted confidence intervals) but the sensitivity did not change. Indeed, a false-positive FDG-PET scan is likely to alert the clinician for the presence of infectious process. ${ }^{[17]}$ Nearly $31 \%$ of the NSCLC clinical presentations are unobtainable as biopsy through conventional means. FDG-PET can help for directed biopsy to increase the potential yield of malignant tissue.

In a retrospective study to assess the accuracy and efficacy of FGD PET-CT in the evaluation of pulmonary nodules, $\mathrm{Li}$ et al. reported $80.2 \%$ sensitivity and $38 \%$ specificity with a calculated positive predictive value of $86 \%$ and overall accuracy of $73.1 \%$. The strength of the study lies in the fact that it was correlated with the histopathological findings after surgical approaches. They also performed a multivariate analysis that helped them to identify risk factors for false negatives; nonsmokers with $<3 \mathrm{~cm}$ lesions in periphery and degree of differentiation of primary tumor false-negative findings with FDG-PET are more likely to be with lesions close to the diaphragm due to undersampling, and partial volume effects ${ }^{[18]}$ with no pleural involvement $(P=0.001)$ predict negative findings of NSCLC. ${ }^{[18]}$ Since the PET acquisition takes longer, misregistration is likely between the two modalities. The grade of tumor also influences the likelihood of uptake within the nodule (low FDG uptake in the lepidic type of adenocarcinoma - the histopathology cannot be predicted 
before imaging), however, FDG-PET has no predictability in terms of outcomes. ${ }^{[19]}$

Therefore, the use of FDG-PET should ideally with these caveats.

Tumor responses are dynamic with a rapidly evolving timeline of genetic mutations. "Liquid biopsies," based on the detection of circulating free DNA (cfDNA) or circulating tumor cells (CTC), have been developed to obviate the need for invasive methods (vide supra). They provide a useful spectrum of oncological evolution to tailor personalized medicine. CTC are selectively enriched by the use of CTC-iChip (using CellSave platform but material needs to be processed within $96 \mathrm{~h}$ ) for identification of allelic mutations. ${ }^{[20]}$ Increased numbers in blood are correlated with poor progression and overall survival and validated using FDG-PET scan for correlation. Cell-free DNA (cfDNA measuring up to $150 \mathrm{bp}$ ) can be analyzed from biobanked plasma. They arise from cells undergoing apoptosis. Its presence reveals mutations and other genetic/ epigenetic phenomenon (amplifications/translocations etc.) but not the transcriptome/proteome. cfDNA has been prospectively validated for EGFR mutation analysis, in the absence of tissue biopsy. For both CTC and cfDNA, the challenges remain for storage, processing, and identification of mutation in the background of excess wild-type DNA. ${ }^{[16]}$

Extracellular genetic material packaged vesicles are known as an exosome. They participate in intercellular messaging and as stable carriers of genetic material from the originating cell. They are shed in excessive numbers $\left(10 \mathrm{e}^{11}\right)$ in plasma, making their identification easier even in stored biosamples. Nanoscale fluorescence-activated cell sorting helps sort out discreet exosomes for further characterization. Despite the obvious advantages of the study of mutations, epigenetic modifications, RNA transcription, and assessment of inflammatory responses, exosomes are unable to give information about phenotypic characteristics of cells.

The use of complementary and newer diagnostic approaches in the identification of pulmonary nodules/masses needs comprehensive elucidation. They have definitely widened the scope for detection (via minimally invasive methods), accurate identification by excluding differentials (improving on the tumor to normal cells ratio by selective enrichment of tumor cells), and treatment monitoring (by improving our understanding of in vivo tumor evolution) of this deadly cancer variant. This has opened new vistas in translational medicine for proper tailoring of targeted therapies. Tissue biopsy remains the "gold standard" despite the obvious limitation of providing just a snapshot of the heterogeneous molecular event. This raises more questions than answers. Is it possible to preselect regional areas that are likely to yield more tumor tissue? How well do we understand the complex biological evolution of the tumor? What firm deductions can be drawn from repeated sampling? Does the liquid biopsy signal the beginning of the end of tissue diagnosis? These burning issues need more definitive and qualitative answers with prospective trials.

\section{Sheh Rawat, Abhishek Purr'}

Department of Radiation Oncology, Dharamshila Cancer Institute, New Delhi, ${ }^{1}$ Department of Radiation Oncology, Fortis Cancer Institute, Mohali, Punjab, India

Address for correspondence: Dr. Abhishek Puri, Fortis Cancer Institute, Phase VIII, Mohali, Punjab, India. E-mail: ajo@abhishekpuri.com

\section{REFERENCES}

1. Rafiemanesh H, Mehtarpour M, Khani F, Hesami SM, Shamlou R, Towhidi F, et al. Epidemiology, incidence and mortality of lung cancer and their relationship with the development index in the world. J Thorac Dis 2016;8:1094-102.

2. Mao Y, Yang D, He J, Krasna MJ. Epidemiology of lung cancer. Surg Oncol Clin N Am 2016;25:439-45.

3. Mitsudomi T. Molecular epidemiology of lung cancer and geographic variations with special reference to EGFR mutations. Transl Lung Cancer Res 2014;3:205-11.

4. Hashim D, Boffetta P, La Vecchia C, Rota M, Bertuccio P, Malvezzi M, et al. The global decrease in cancer mortality: Trends and disparities. Ann Oncol 2016;27:926-33.

5. Sutedja G. New techniques for early detection of lung cancer. Eur Respir J Suppl 2003;39:57s-66s.

6. Zaric B, Stojsic V, Sarcev T, Stojanovic G, Carapic V, Perin B, et al. Advanced bronchoscopic techniques in diagnosis and staging of lung cancer. J Thorac Dis 2013;5 Suppl 4:S359-70.

7. Neal JW. Histology matters: Individualizing treatment in non-small cell lung cancer. Oncologist 2010;15:3-5.

8. Moreira AL, Thornton RH. Personalized medicine for non-small-cell lung cancer: Implications of recent advances in tissue acquisition for molecular and histologic testing. Clin Lung Cancer 2012;13:334-9.

9. Kim L, Tsao MS. Tumour tissue sampling for lung cancer management in the era of personalised therapy: What is good enough for molecular testing? Eur Respir J 2014;44:1011-22.

10. Arnedos M, Soria JC, Andre F, Tursz T. Personalized treatments of cancer patients: A reality in daily practice, a costly dream or a shared vision of the future from the oncology community? Cancer Treat Rev 2014;40:1192-8.

11. Zheng M. Classification and pathology of lung cancer. Surg Oncol Clin N Am 2016;25:447-68.

12. Truong MT, Ko JP, Rossi SE, Rossi I, Viswanathan C, Bruzzi JF, et al. Update in the evaluation of the solitary pulmonary nodule. Radiographics 2014;34:1658-79.

13. Zhang M, Zhuo N, Guo Z, Zhang X, Liang W, Zhao S, et al. Establishment of a mathematic model for predicting malignancy in solitary pulmonary nodules. J Thorac Dis 2015;7:1833-41.

14. Swensen SJ, Silverstein MD, Edell ES, Trastek VF, Aughenbaugh GL, Ilstrup DM, et al. Solitary pulmonary nodules: Clinical prediction model versus physicians. Mayo Clin Proc 1999;74:319-29.

15. Gould MK, Donington J, Lynch WR, Mazzone PJ, Midthun DE, Naidich DP, et al. Evaluation of individuals with pulmonary nodules: 
When is it lung cancer? Diagnosis and management of lung cancer, $3^{\text {rd }}$ ed: American College of Chest Physicians evidence-based clinical practice guidelines. Chest 2013;143 5 Suppl: e93S-120S.

16. Brock G, Castellanos-Rizaldos E, Hu L, Coticchia C, Skog J. Liquid biopsy for cancer screening, patient stratification and monitoring. Transl Cancer Res 2015;4:280-90.

17. Deppen SA, Blume JD, Kensinger CD, Morgan AM, Aldrich MC, Massion PP, et al. Accuracy of FDG-PET to diagnose lung cancer in areas with infectious lung disease: A meta-analysis. JAMA 2014;312:1227-36.

18. Li S, Zhao B, Wang X, Yu J, Yan S, Lv C, et al. Overestimated value of (18) F-FDG PET/CT to diagnose pulmonary nodules: Analysis of 298 patients. Clin Radiol 2014;69:e352-7.

19. Padma S, Sundaram PS, George S. Role of positron emission tomography computed tomography in carcinoma lung evaluation. J Cancer Res Ther 2011;7:128-34.

20. Karachaliou N, Mayo-de-Las-Casas C, Molina-Vila MA, Rosell R. Real-time liquid biopsies become a reality in cancer treatment. Ann Transl Med 2015;3:36.
This is an open access article distributed under the terms of the Creative Commons Attribution-NonCommercial-ShareAlike 3.0 License, which allows others to remix, tweak, and build upon the work non-commercially, as long as the author is credited and the new creations are licensed under the identical terms.

\begin{tabular}{|l|c|}
\hline \multicolumn{3}{|c|}{ Access this article online } \\
\hline \multirow{2}{*}{ Website: } & Quick Response Code \\
www.asjo.in & \\
\hline & DOI: \\
10.4103/2454-6798.209337
\end{tabular}

How to cite this article: Rawat S, Puri A. Lung cancer: Is biopsy mandatory. Asian J Oncol 2017;3:1-4. 